\title{
A novel TGF-beta/Smad signaling inhibitor ameliorates bleomycin-induced skin fibrosis
}

Minoru Hasegawa, Vu Huy Luong, Takenao Chino, Noritaka Oyama, (Department of Dermatology, Fukui Univ., Japan)

Yoko Sasaki, Dai Ogura, Sin-ichiro Niwa (Link Genomics, Co., Ltd., Japan)

Yoshinari Okamoto, Masami Otsuka (Department of Bioorganic Medicinal Chemistry, Kumamoto Univ., Japan) Mikako Fujita (Research Institute for Drug Discovery, Kumamoto Univ., Japan)

Fukui University

Hironobu Ihn (Department of Dermatology and Plastic Surgery, Kumamoto Univ., Japan)

\section{Introduction}

Systemic sclerosis (SSc) is a chronic autoimmune disease characterized by progressive fibrosis of skin and internal organs. TGF- $\beta /$ Smad signaling is well known to play a crucial role in the pathogenesis of SSc. Recently, we developed a novel compound, HPH-15, which antagonizes TGF$\beta /$ Smad signaling.

\section{Objective}

The purpose of the current study was to clarify the effects of this drug in a preclinical model of SSc and in human skin fibroblasts.

- The antifibrotic properties of HPH-15 were examined in a bleomycin-induced skin fibrosis mouse model.

- The effects of HPH-15 on migration, proliferation, expression of extracellular matrix components, and TGF- $\beta$ signaling in human dermal fibroblasts were analyzed.

\section{Materials and Methods}

C57BL/6J: female, 10-12 weeks, $\mathbf{n}=$ 3-5

Bleomycin (BLM): subcutaneous (150 $\mu \mathrm{g} /$ day)

HPH-15: oral ( $100 \mathrm{mg} / \mathrm{kg}$ daily)

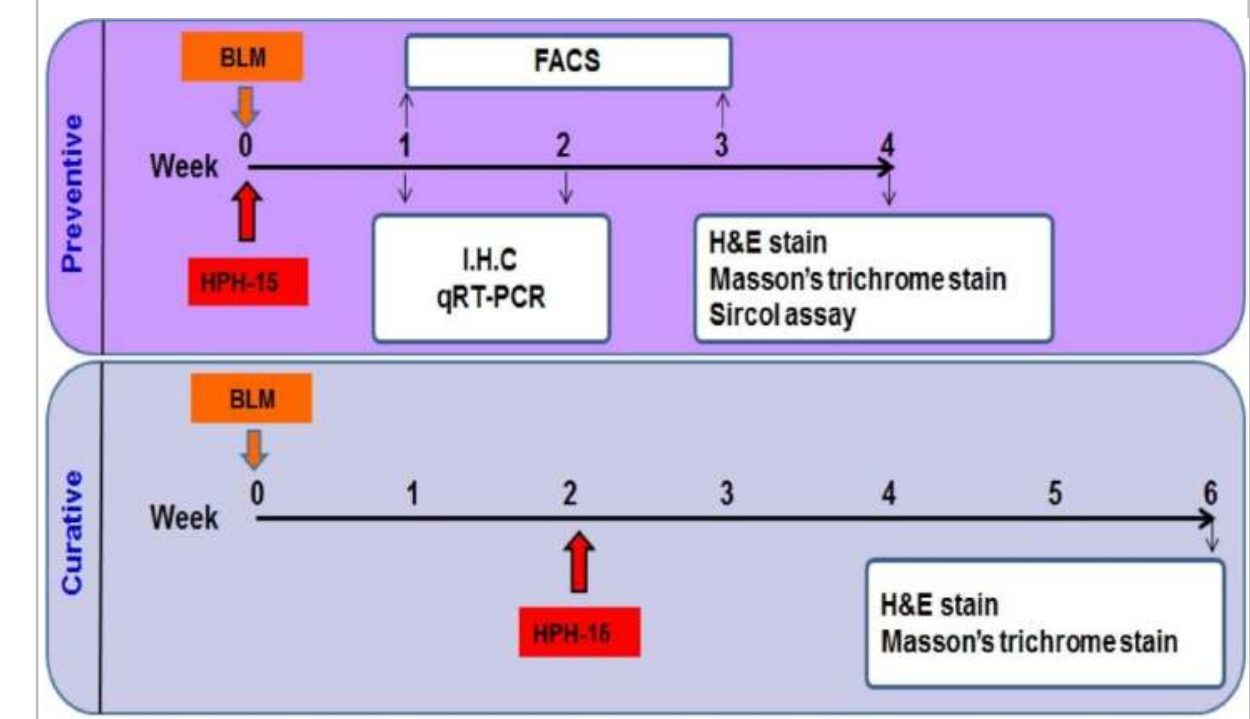

Human dermal fibroblasts

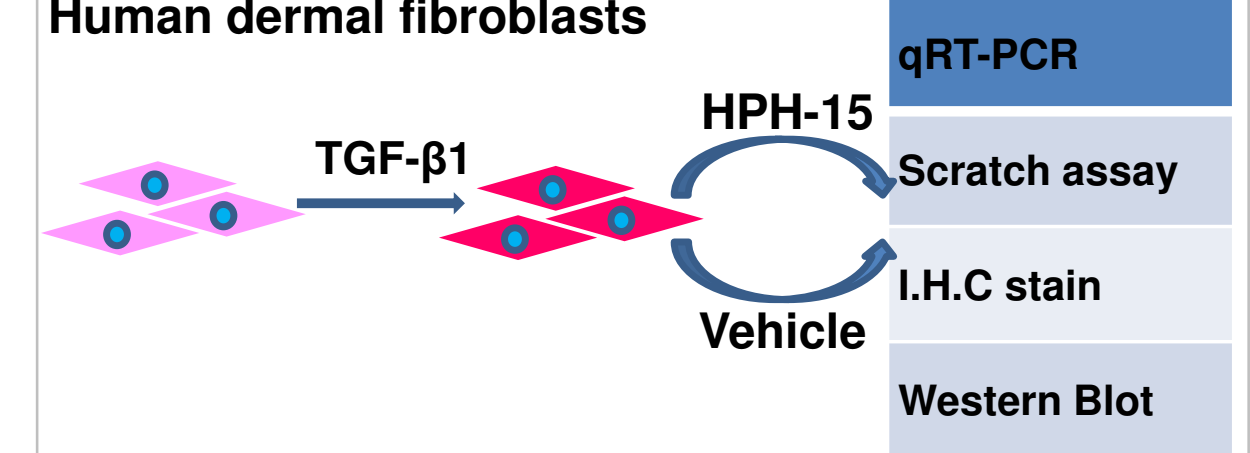

\section{Results}

HPH-15 treatment ameliorated dermal thickening in the BLM-injected skin
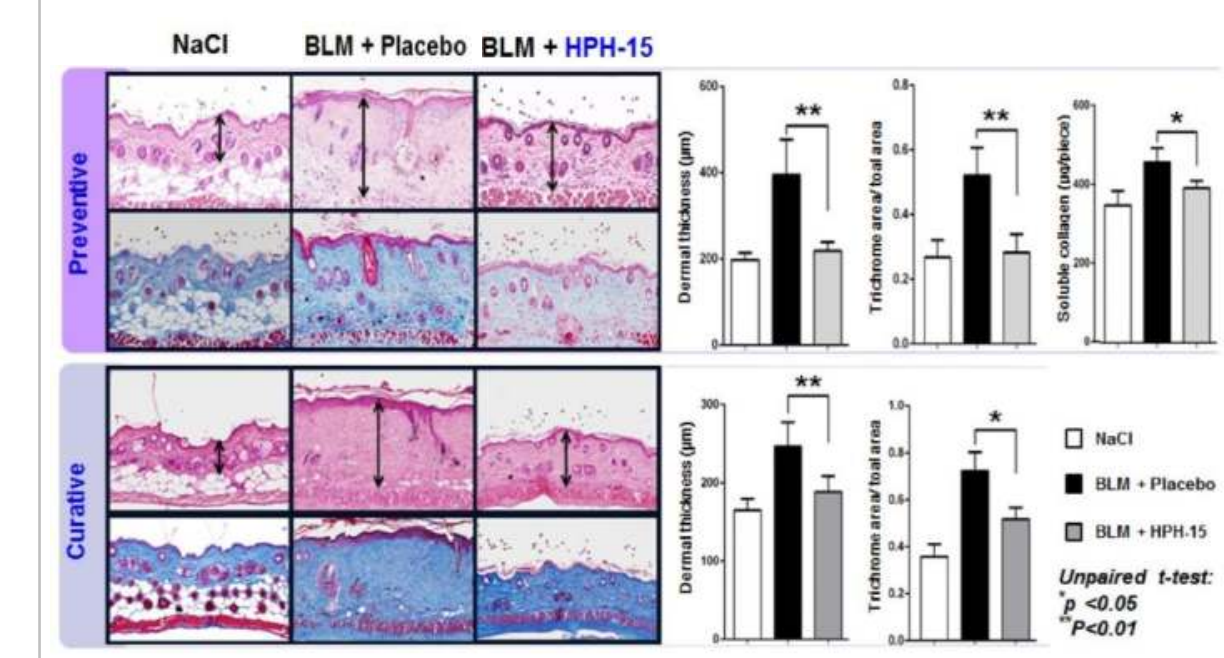

$\mathrm{HPH}-15$ treatment reduced the number of $\mathrm{CD}^{+} \mathrm{T}$ cells and $\mathrm{F} 4 / 80^{+}$macrophages in BLM-injected skin

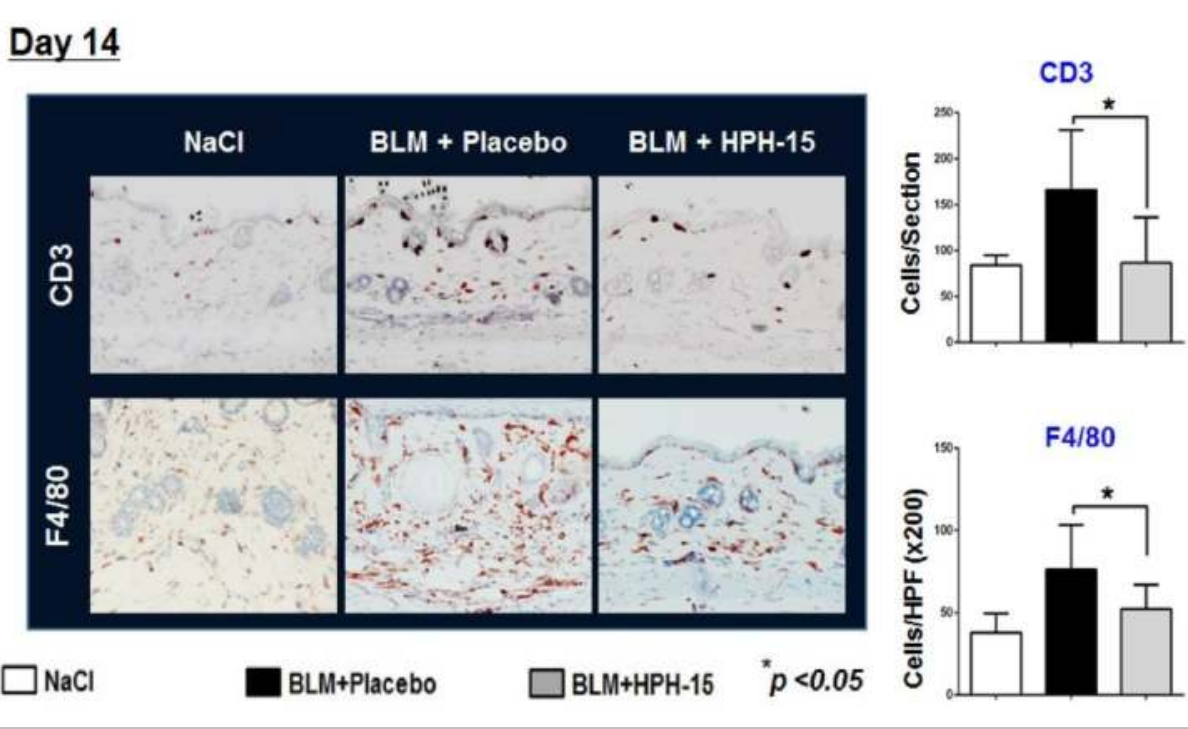

$\mathrm{HPH}-15$ treatment reduced inflammatory macrophages in the early stage and profibrotic M2 macrophages in the late stage

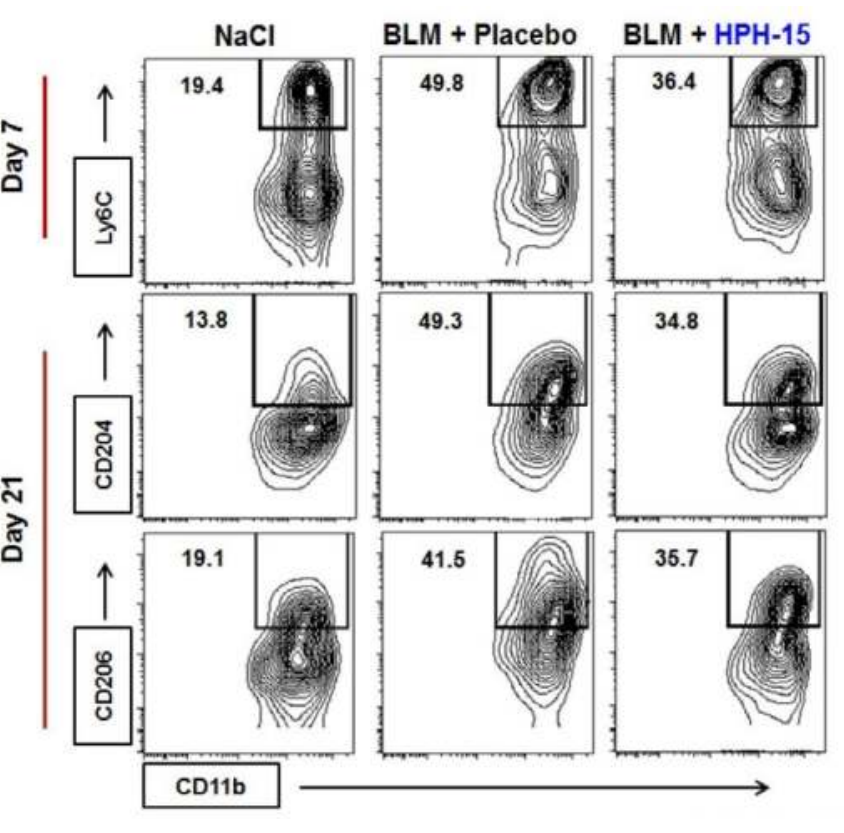

$\square \mathrm{NaCl}$
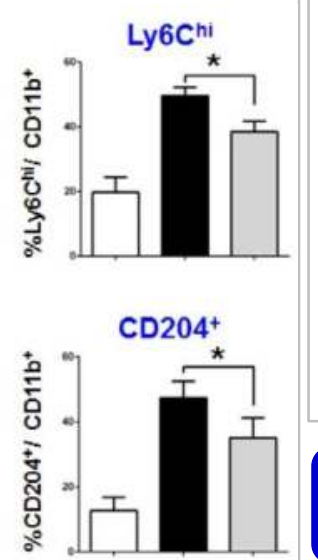

$\mathrm{CD}^{206^{+}}$

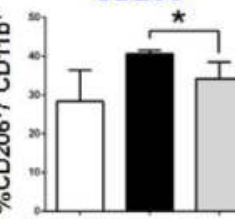

${ }^{*} p<0.05$

HPH-15 inhibited the mRNA levels of M2 macrophage markers, not M1 macrophage marker

Skin-day 14 Arg1

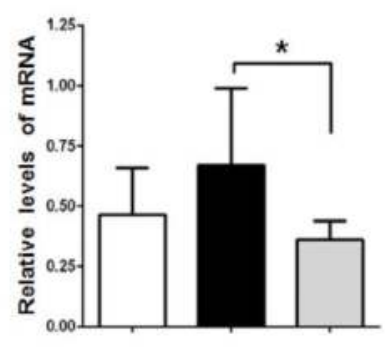

$\square \mathrm{NaCl}$
Ym1

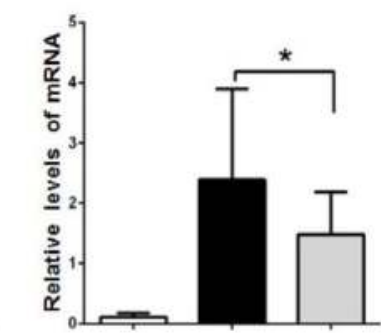

BLM+Placebo
iNOS

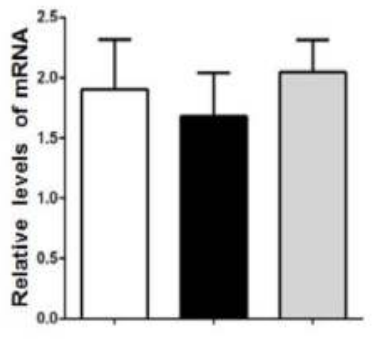

$p<0.05$
HPH-15 recovered the mRNA levels of Fil1 and KLF5, transcription factor that repress collagen synthesis Skin-day 14
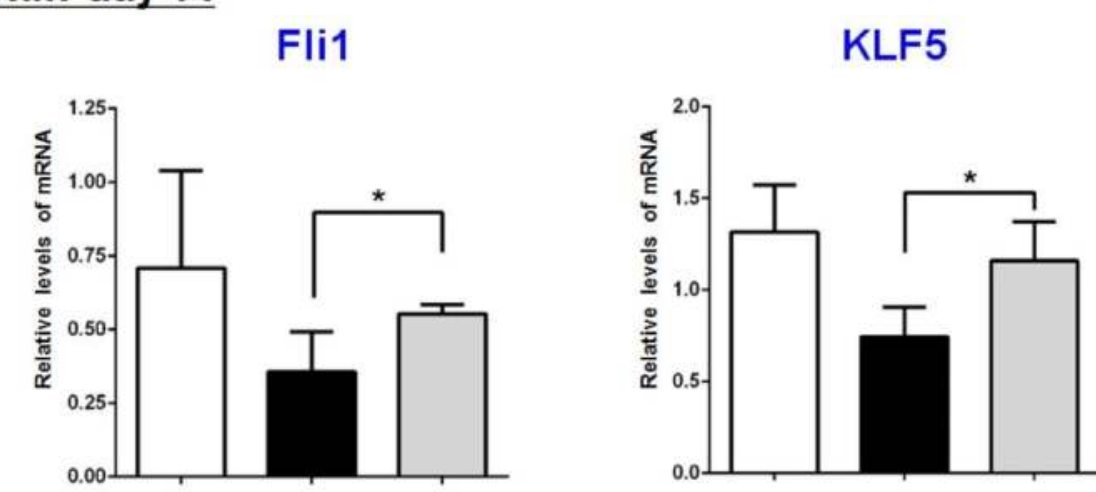

$\square \mathrm{NaCl}$

BLM+Placebo

$\square \mathrm{BLM+HPH}-15$

HPH-15 inhibited fibrotic responses induced by TGF- $\beta$ in cultured human dermal fibroblasts Col1a2

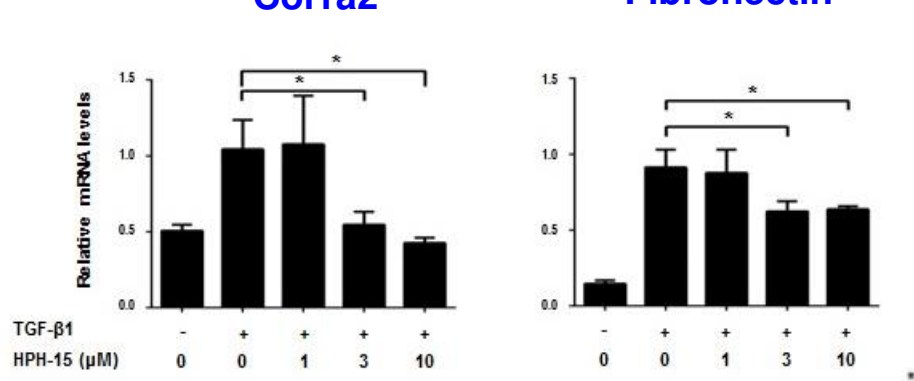

HPH-15 inhibited fibroblasts migration and/or proliferation
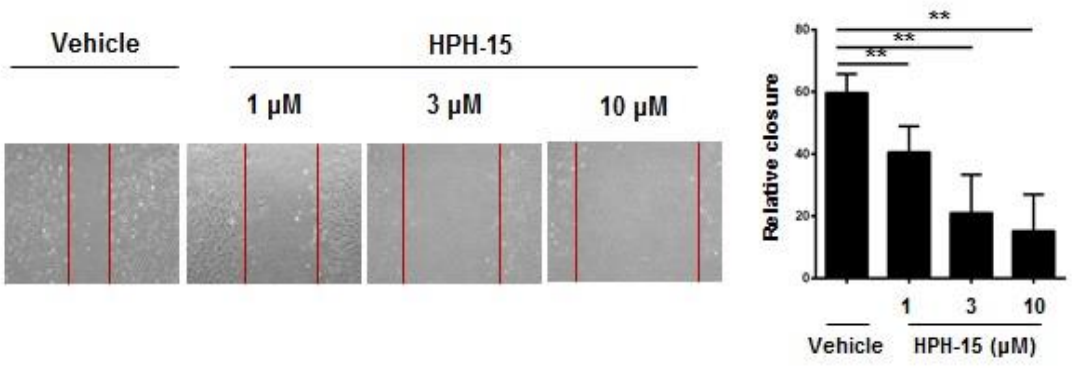

$p<0.05$

HPH-15 antagonizes the over-phosphorylation of Smad3 in the mouse skin and cultured human dermal fibroblasts
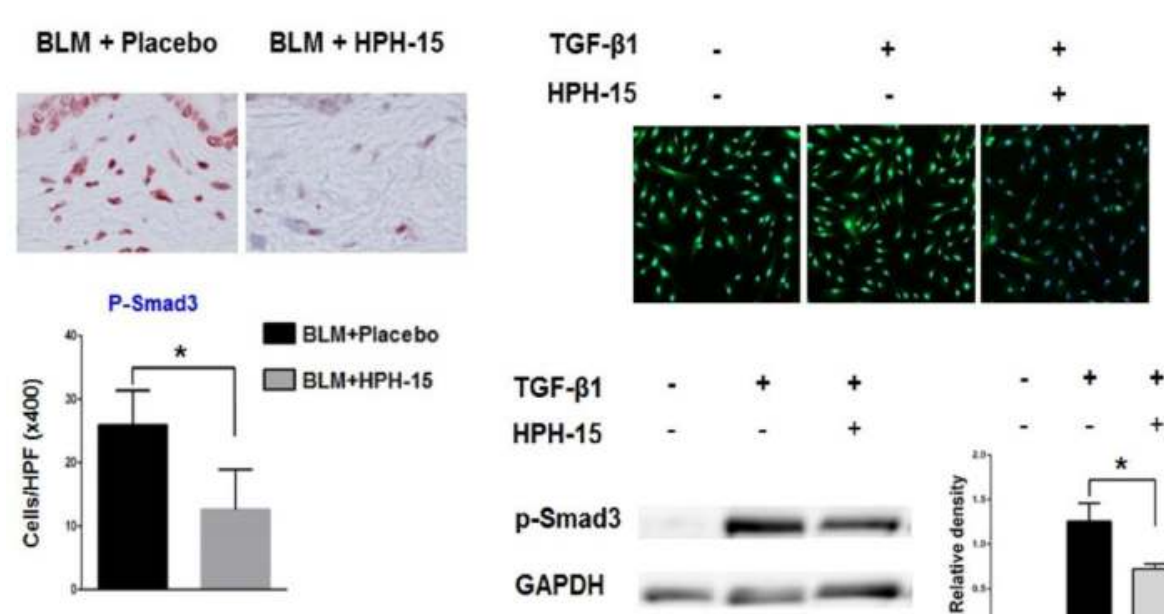

TGF-

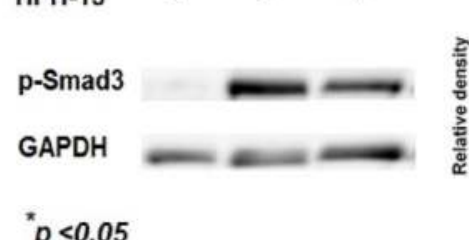

$p<0.0$

\section{Summary}

Administration of HPH-15 demonstrated:

1. Suppression of skin thickening and collagen accumulation in the BLM-induced skin fibrosis mouse model.

2. Reduction of increased numbers of macrophages and $T$ cells in the BLM-injected skin.

3. Decreased dermal infiltration of proinflammatory macrophages (CD11b+Ly6C ${ }^{\text {hi}}$ ) and M2 profibrotic macrophages (CD11b+CD204+ or CD11b+CD206 $\left.{ }^{+}\right)$ during the early and late stages, respectively.

4. Augmentation of the mRNA levels of Fli1 and KLF5, and reduced M2 macrophage markers in the BLM-injected skin.

5. Inhibition of fibrotic responses induced by TGF- $\beta$ in cultured human fibroblasts.

6. Antagonizing Smad3 phosphorylation in the mouse skin and cultured human dermal fibroblasts.

\section{Conclusion}

Our study clearly shows that HPH-15 inhibits TGF$\beta /$ Smad signaling and fibrogenic activity of human skin fibroblasts in vitro and attenuates skin inflammation and subsequent fibrosis in a mouse model of SSc. Since the findings of this early preclinical study demonstrate several advantages of HPH-15, including the fact that it is an orally active agent with an excellent safety profile, $\mathrm{HPH}-15$ shows potential as a strong candidate for SSc clinical trials. 